\title{
Tekil Spektrum Analizi ve Uzun-Kısa Süreli Bellek Ağları ile Nehir Akım Tahmini*
}

\author{
Levent Latifoğlu ${ }^{1 * *}$, Kazım Bekir Nuralan ${ }^{2}$ \\ ${ }^{1}$ Erciyes Üniversitesi, Mühendislik Fakültesi, İnşaat Müehndisliği Bölümü, Kayseri, Türkiye (ORCID: 0000-0002-2837-3306) \\ ${ }^{2}$ Erciyes Üniversitesi, Mühendislik Fakültesi, İnşaat Müehndisliği Bölümü, Kayseri, Türkiye (ORCID: 0000-0001-5764-805X)
}

(Konferans Tarihi: 5-7 Mart 2020)

(DOI: 10.31590/ejosat.araconf49)

ATIF/REFERENCE: Latifoğlu, L. \& Nuralan, K. B. (2020). Tekil Spektrum Analizi ve Uzun-Kısa Süreli Bellek Ağları ile Nehir Akım Tahmini. Avrupa Bilim ve Teknoloji Dergisi, (Özel Sayı), 376-381.

$\ddot{O} z$

Su yapılarının planlanması ve yönetiminde nehir akım tahminleri önemli bir yere sahiptir. Lineer olmayan ve durağan olmayan karaktere sahip nehir akım verilerinin doğru tahmini zorlu bir problemdir. Son yıllarda veri tabanlı teknikler, nehir akım problemlerinde yoğun olarak kullanılmaktadır. Önerilen çalışmada popüler olarak kullanılmaya başlanan Derin Sinir Ağlarından Uzun - Kısa Süreli Bellek (Long-Short Term Memory, LSTM) Ağları ile nehir akım tahmini gerçekleştirilmiştir. Tahmin performansını artırmak üzere zaman serilerinin analizinde önemli bir yer tutan Tekil Spektrum Analizi (TSA) kullanılarak alt bant verileri elde edilmiştir. Nehir akım tahmin verisine ait TSA alt bant verilerinin LSTM ağları ile tahmini sonucu bir ileri adım tahmin çalışması gerçekleştirilmiştir. Önerilen TSA-LSTM modeli kullanılarak 0.0021 Ortalama Karesel Hata (MSE) değeri, 0.0361 Ortalama Mutlak Hata (MAE) değeri ve 0.9710 Korelasyon (R) değeri ile yüksek performanslı tahmin verisi elde edilmiştir.

Anahtar Kelimeler: Nehir akım tahmini, Derin Sinir Ağları, Kısa-Uzun Bellek Ağları, Tekil Spektrum Analizii.

\section{Forecasting Of Stream Flow Usıng Singular Spectrum Analysis And Long-Short Term Memory Networks}

\begin{abstract}
Stream flow estimation has an important role in the planning and management of water resources. Accurate estimation of stream flow data, that is characterised ny non-linear and non-stationary, is a challenging problem. In recent years, data-based techniques have been used extensively in forecasting of stream flow. In this study, stream flow estimation was made with the Long-Short Term Memory (LSTM) Networks from Deep Neural Networks, which were used as popular. Subband data was obtained by using Single Spectrum Analysis (SSA), which plays an important role in the analysis of time series in order to increase the forecast performance. As a result of estimation of SSA subband data of stream flow forecasting data with LSTM network, one ahead forecasting study was carried out. Using this proposed SSA-LSTM model, high performance forecasted data was obtained with 0.0021 Mean Square Error (MSE) value, 0.0361 Mean Absolute Error (MAE) value and 0.9710 Correlation (R) coefficient value.
\end{abstract}

Keywords: Stream flow forecasting, Deep Neural Networks, Long-Short Term Neural Networks, Singular Spectrum Analysis.

\footnotetext{
* Bu makale International Conference on Access to Recent Advances in Engineering and Digitalization (ARACONF 2020) de sunulmuştur.

** Sorumlu Yazar: Erciyes Üniversitesi, Mühendislik Fakültesi, İnşaat Mühendisliği Bölümü, Kayseri, Türkiye, ORCID: 0000-0002-2837-3306, latifoğlu@erciyes.edu.tr
} 


\section{Giriş}

Nehir akımlarının doğru ve hassas tahmini rezervuar yönetiminde, risk değerlendirilmesinde, kuraklık tahmininde, taşkın ötelemede, afet yönetiminde, su planlaması ve yönetiminde önemli role sahiptir. Tahmin modelleri süreç tabanlı ve veri tabanlı olmak üzere temel olarak iki kategoride incelenmektedir [1].

Süreç tabanlı tekniklerde süreçler temel olarak iki aşamada incelenmektedir. İlk aşama matematiksel modellerin oluşturulması, ikinci aşama ise sayısal çözümlerin eldesidir. Matematiksel modelleme aşamasında, işlem matematiksel denklemler ile tanımlanmaktadır. Daha sonra, bu denklemlerin doğru ve verimli bir sayısal çözümü gerçekleştirilmektedir. Bu modellerde ilgili süreci teorik olarak anlaşılması gerekmektedir ve yüksek miktarda veri gereksinimi ile birlikte yaklaşımlar içeren varsayımlar ortaya konmaktadır [2]. Sürece dayalı modelleri geliştirmek için, süreci tanımlayan doğru verilerle birlikte sürecin çok iyi anlaşılması gerekmektedir. Modellenmesi gereken süreç hakkında bilgi eksikliği durumda sürece dayalı modellerin gerçekleştirilmesi mümkün değildir. Öte yandan, veriye dayalı modeller ampirik olup, gözlemlere dayanmaktadır, basit ve uygulanması daha kolaydır. Ayrıca süreç ile ilgili fiziksel bilgi gerektirmemektedir. Veri tabanlı teknikler temel olarak kara kutu modeller olarak tanımlanmaktadır. Veri tabanlı teknikler arasında işlemsel zeka ve makine öğrenme yöntemleri, sinir ağları, bulanık kural tabanlı sistemler ve genetik algoritmalar ile bunların kombinasyonu yaygın olarak kullanılmaktadır. [3]. Tahmin çalışmalarında istatistiksel olarak veriye dayalı teknikler 1970 li yıllarda ortaya konmuştur. Bu teknikler lineer yaklaşımlar üzerine kurulu olup, parametrik Özbağılımlı Hareketli ortalama (ÖBHO/ARMA) ve Özbağılımlı İntegralli Hareketli Ortalama (ÖBİHO/ARIMA) gibi modeller ve bu modellerin farklı versiyonları nehir akım tahmininde kullanılmaktadır [4-7]. Nehir akım verileri doğası gereği lineer olmayan ve durağan olmayan karakterdedir. $\mathrm{Bu}$ verilerin tahmini ve simülasyonu için son yıllarda makine öğrenme teknikleri yaygın olarak kullanılmaktadır. .Makine öğrenme tekniklerinden Yapay Sinir Ağları (YSA), Karar Destek Makinaları (KDM), Genetik programlama (GP), Adaptif A $\breve{g}$ Yapısına Dayalı Bulanık Çıkarım Sistemi (ANFIS) gibi yöntemler lineer olmayan nehir akım verilerinin tahmininde kayda değer sonuçlar ortaya koymuştur [8-15]. Son yıllarda, beynin yapısından ve işlevinden ilham alan yapay sinir ağları tabanlı algoritmalarla ilgili bir makine öğrenmesinin alt alanı olan Derin öğrenme yaklaşımı tahmin çalışmalarında kullanılmaktadır. [16-18]. Derin öğrenme, çok katmanlı modellerle yapılan yapay öğrenme olup, Yapay Sinir Ağlarındaki gizli katmanın sayısının artırılmış ve geliştirilmiş halidir. Tekrarlayan Yapay Sinir Ağları (Recurrent Neural Network, RNN) bir çeşit YSA modeli olup bir döngü oluşturarak geçmiş bilgilerin kullanılmasını sağlamaktadır. Bu sayede önceki zamanın bilgileri kullanılabilmektedir ve yeni bilgi, eski bilgi kullanılarak elde edilerek, sınıflandırma yapılabilmektedir [19]. Uzun-Kısa Süreli Bellek Ağları uzun dizilerle gösterilen veriler üzerinde eğitim ve hafızayı koruma problemine yönelik ortaya konulan bir RNN mimarisidir. LSTM mimarisinin RNN mimarisine göre en temel avantajları; sıfırlanan gradyan problemine çözüm oluşturabilmesi ve girdilerin unutulmadan saklanabilmesi aracılığıyla bilgi kaybını engelleyebilmesidir. Bu nedenle LSTM mimarisi yaygın olarak kullanılmaya başlanmıştır [20].

Nehir akım verilerinin ön işleme tabi tutulması, veriye dayalı modellerin tahmin performansını artırmaktadır. Literatürde, Ampirik Kip Ayrışım (AKA), Ayrık Dalgacık Dönüşümü ve Tekil Spektrum Analizi yöntemleri verilerin ön işlenmesi aşamasında kullanılarak veriye dayalı modellerle tahmin gerçekleştiren çalışmalar bulunmaktadır [21-24]. Ancak bu çalışmalar arasında TSALSTM modeli kullanarak nehir akım tahmini gerçekleştiren çalışmaya rastlanmamıştır.

Bu çalışmada, TSA-LSTM modeli kullanarak gerçekleştirilen nehir akım tahmini ile önerilen modelin tahmin performansı analiz edilmiştir.

\section{Materyal ve Metot}

\subsection{Tahmin Çlaışmalarında Kullanılan Hidrolojik Veriler}

Güney Marmara Bölgesinde Susurluk havzasında yer alan Simav çayının mansap kısmını teşkil eden Kocadere üzerindeki Elektrik İşleri Etüt İdaresine (EİEI) ait 317 nolu akım gözlem istasyonundan alınan ortalama aylık akım verileri tahmin çalışmasında kullanılmıştır [25]. Nehir ortalama aylık akım verileri 1965-2011 yılları arasına ait olup, verilerin kaydedildiği havza alanına ait harita Şekil 2. de verilmektedir.

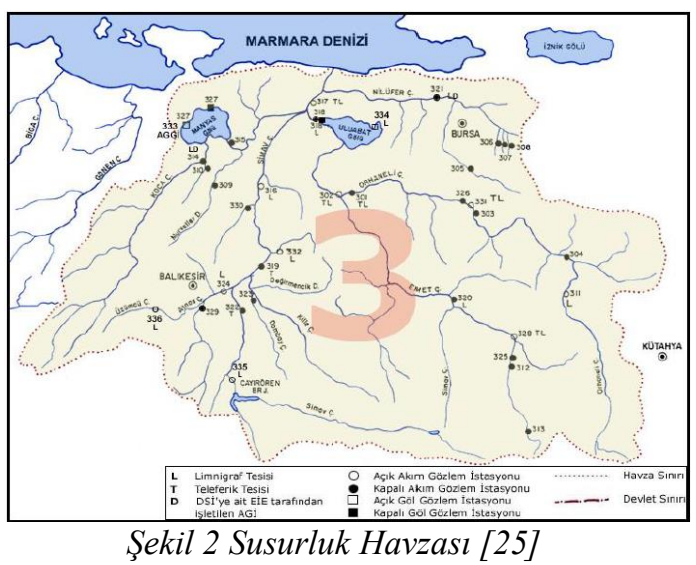


Tahmin çalışmasında akım verilerinin \%70'i eğitim verisi \%30’u ise test verisi olarak kullanılmıştır. Tahmin çalışmasında kullanılan normalize eğitim ve test verileri Şekil 3 te görülmektedir.
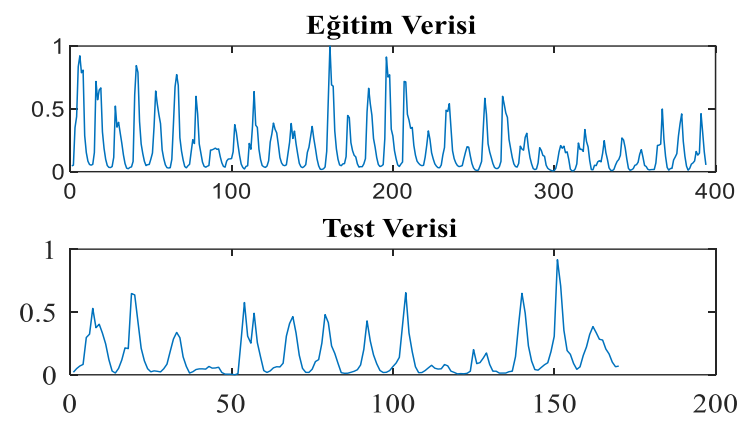

Şekil 3 Tahmin çalışmasında kullanılan eğitim ve test verileri

\section{2. Ön İşleme Ve Tekil Spektrum Analizi}

Yarı parametrik ve analizde ön koşul gerektirmeyen bir yöntem olan TSA, zaman serisi sinyalinin Küçük Aralıklara Gömülmesi işlemi, Tek Değerli Ayrıştırma ile özvektör üçlüsünün eldesi, Gruplandırma ve Diyagonal Ortalama işlemi adımlarından oluşmaktadır. TSA'inde sinyallerin zaman aralıklarına gömülmesi ve tek değerli ayrıştırma adımları Ayrıştırma aşamasını, gruplandırma ve diyagonal ortalama işlemleri ise Yeniden Yapılandırma aşamasını oluşturmaktadır. TSA için detaylı bilgi 26 nolu referansta yer almaktadır [26].

Bildiri çalışmasında nehir akım verileri TSA ile iki adet alt banda ayrıştırılmışıtır. TSA'inde gömme aşamasında akım verilerinin periyodik yapı benzerliğinden dolayı pencere uzunluğu 12 olarak belirlenmiştir. Birinci alt bant verisi 1-5 özdeğerine göre 2. alt bant verisi ise 6-12 özdeğerine göre yeniden gruplandırılmıştır. Şekil 4 te eğitim ve test aşamasında kullanılan TSA alt bant verileri görülmektedir.

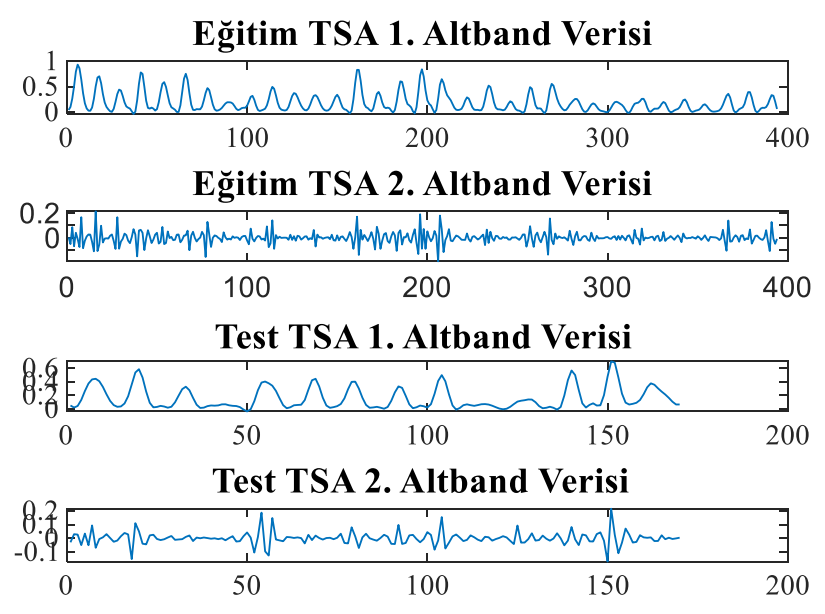

Şekil 4 Tahmin çalışmasinda kullanılan TSA altbant verileri

\subsection{Uzun-Kısa Süreli Bellek Ağları}

RNN mimarisinin bir türü olan LSTM ilk defa Hochreiter \& Schmidhuber (1997) tarafindan ortaya konmuştur ve ilerleyen zamanlarda birçok kişi tarafindan geliştirilmiştir [27].

RNN mimarisinden farklı olarak LSTM mimarisinde, uzun süre bir önceki girdiyi hatıllamak için kullanılan ve hafıza hücreleri olarak adlandırılan özel gizli birimler bulunmaktadır. Unutma, Giriş, Güncelleme ve Çıkış kapısı katmanından oluşan LSTM mimarisi, bir girdinin önemli olup olmadı̆̆ını belirleyerek kaydedilmesine karar veren kapılar içerir [28]. Şekil 5 'te LSTM yapıSı görülmektedir. 


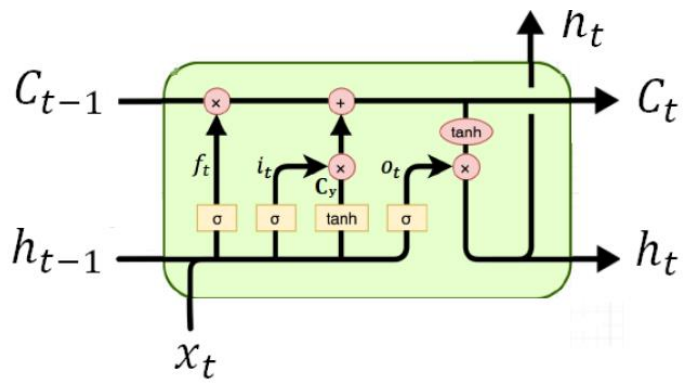

Şekil 5. LSTM Yapısi [28]

\subsection{Performans Parametreleri}

Hidrolojik verilerin tahmini çalışmalarında tahmin performansını değerlendirmek üzere yaygın olarak kullanılan parametrelerden Ortalama Mutlak Hata (MAE), Ortalama Karesel Hata (MSE), ve Korelasyon katsayısı (R) bu çalışmada kullanılmıştır.

\subsubsection{Ortalama Karesel Hata}

Veri setinin gözlenen ve tahmin edilen değerlerinin farkının karelerinin toplamının toplam veri sayısına bölünmesiyle elde edilen değerdir.

\subsubsection{Ortalama Mutlak Hata}

Veri setinin gözlenen ve tahmin edilen değerleri farkının mutlak değer toplamının veri sayısına bölünmesiyle elde edilmektedir.

\subsubsection{Korelasyon Katsayıs}

İki veri seti arasında doğrusal bir ilişki bulunup bulunmadığının yönünün ve derecesinin bir göstergesidir. R değeri Pearson Korelasyon Katsayısı olarak ta isimlendirilmektedir ve Eşitlik 1 deki formülle hesaplanmaktadır.

$R=\frac{1}{N-1} \sum_{i=1}^{N}\left(\frac{X_{i}-\mu_{X}}{\sigma_{X}}\right)\left(\frac{Y_{i}-\mu_{Y}}{\sigma_{Y}}\right)$

Verilen eşitlikte $\mu_{X}$ ve $\sigma_{X} \mathrm{X}$ veri setinin ortalama ve standart sapmasını $\mu_{Y}$ ve $\sigma_{Y} \mathrm{Y}$ veri setinin ortalama ve standart sapmasını göstermektedir.

\section{Araştırma Sonuçları ve Tartışma}

Önerilen çalışmada TSA-LSTM modelinin performansı LSTM modeli ile karşılaştırılmıştır. Tahmin çalışması modelleri için bir adet giriş uygulanmıştır. Bir ileri tahmin işlemi gerçekleştirilen bu çalışmada modellerin tahmin performansı analiz edilmiştir.

\subsection{Uzun-Kısa Süreli Bellek Ağları}

Gerçekleştirilen çalışmada normalize nehir akım verileri üzerinde bir ön işlem yapılmadan LSTM Modeli ile bir ileri tahmin işlemi gerçekleştirilmiştir. Derin Sinir Ağı mimarisinin oluşturulması aşamasında giriş katmanı (Sequence Input Layer), LSTM katmanı, Tam Bağlantılı Katman (Fully Connected Layer) ve Regresyon katmanı kullanılmıştır. Ağın eğitimi aşamasında maksimum devir (epoch ) sayısı 200, başlangıç öğrenme oranı 0.002, öğrenme oranı düşme aralığı 200, öğrenme oranı düşme faktörü ise 0.1 olarak belirlenmiştir.

Ağın eğitimi için 'Adam', 'Stochastic Gradient Descent with Momentum (SGDM)' ve 'Root Mean Square Propogation (RMSPROP)' optimizasyon algoritması kullanılarak performansları karşılaştırılmıştır. Bir ileri tahmin sonucu elde edilen performans parametre değerleri Tablo 1 de görülmektedir.

Tablo 1. SGDM, RMSPROP ve Adam optimizasyon algoritmalarının tahmin çalışmasındaki performans değerleri

\begin{tabular}{l|c|c|c}
\hline & MSE & MAE & R \\
\hline \multicolumn{5}{|c|}{ LSTM modeli } \\
\hline RMSPROP & 0.0112 & 0.0737 & 0.7892 \\
\hline Adam & 0.0148 & 0.0966 & 0.7923 \\
\hline
\end{tabular}

Tablo 1 incelendiğinde tahmin çalışmasında en iyi performansın ADAM optimizasyon algoritması ile elde edildiği görülmüştür.

Ayrıca Şekil 6 da gözlem ve ADAM optimizasyon algoritması kullanılan LSTM modeli ile elde edilen tahmin verileri yer almaktadir. 


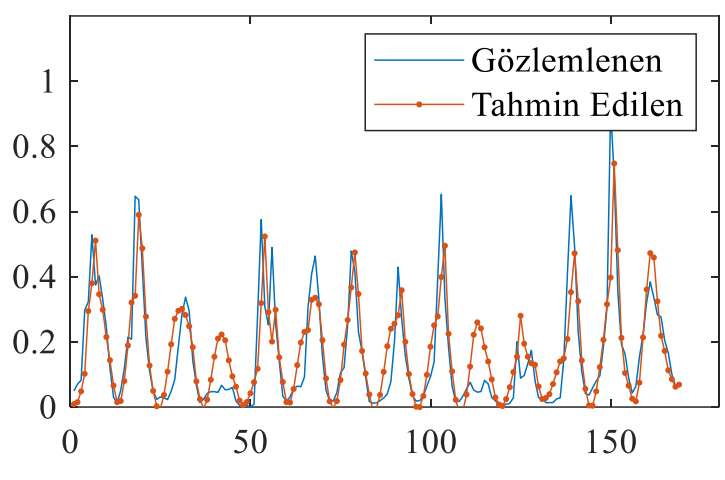

Şekil 6 LSTM Modeli ile bir ileri tahmin grafiği

\subsection{TSA - LSTM Modeli}

Çalışmanın bu aşamasında normalize eğitim ve test nehir akım verileri TSA kullanılarak iki alt banda ayrıştırılmıştır. 1. alt bant bileşeni 1-5. öz değerlere göre elde edilmiş olup verinin eğilim bilgisini verirken 2. alt bant bileşeni ise veride küçük genlikli değişimleri ortaya konmaktadır. Alt bantların tahmininde LSTM modelinde olduğu gibi benzer yapı kullanılmıştır. İki adet Derin Sinir Ağı giriş katmanı, LSTM katmanı, Tam Bağlantılı Katman ve Regresyon katmanından oluşmaktadır. Ağın eğitimi aşamasında da LSTM modelinde olduğu gibi aynı parametreler kullanılmıştır. Optimizasyon algoritması olarak Adam optimizasyon algoritması kullanılmıştır. TSA-LSTM modeli ile bir ileri tahmin performans değerleri Tablo 2 de görülmektedir.

Tablo 2. LSTM ve TSA_LSTM Modeli ile elde edilen tahmin performans değerleri

\begin{tabular}{|c|c|c|c|}
\hline & MSE & MAE & $\mathbf{R}$ \\
\hline \multicolumn{4}{|c|}{ LSTM modeli } \\
\hline 1 giriş-1 çıkıls & 0.0098 & 0.0672 & 0.8168 \\
\hline \multicolumn{4}{|c|}{ TSA-LSTM Modeli } \\
\hline 1 giriş-1 çıkıls & 0.0021 & 0.0361 & 0.9710 \\
\hline
\end{tabular}

Tablo 2 incelendiğinde TSA-LSTM modelinin performansının LSTM modeline göre daha iyi olduğu görülmektedir. Ayrıca Şekil 7 de gözlem ve TSA-LSTM modeli ile elde edilen tahmin verileri yer almaktadır.

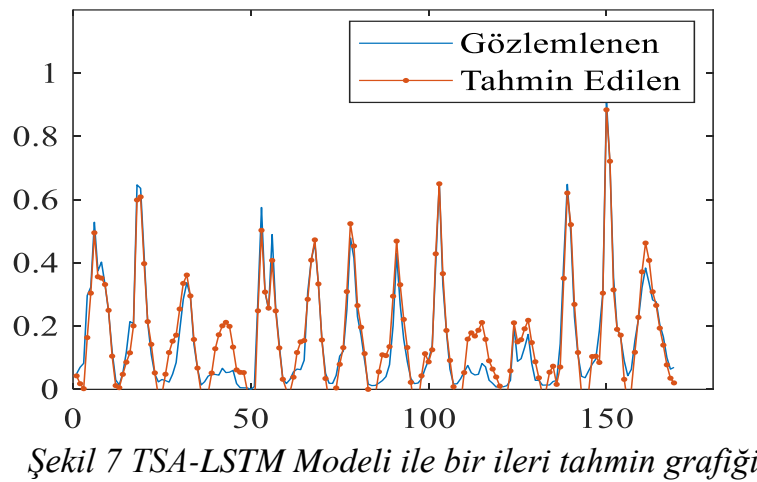

\section{Sonuç}

Bu çalışmada bir Derin Sinir Ağı olan LSTM ağları kullanarak aylık nehir akım verilerinin tahmini gerçekleştirilmiştir. Aylık nehir akım verilerine TSA ile uygulanan ön işlemin tahmin performansına etkisi analiz edilmiştir. LSTM ağı SGDM, RMSPROP ve Adam optimizasyon algoritmaları kullanılarak eğitilmiştir. Ağın eğitimi neticesinde en iyi performans Adam optimizasyon algoritması ile elde edilmiştir. LSTM modeli kullanarak elde edilen bir ileri zamanlı tahmin çalışmasında MSE değeri 0.0098. MAE değeri 0.0672 ve R değeri 0.8168 olarak elde edilmiştir. Bir veri içerisindeki örüntüyü belirleyerek, birbiri ile ilişkili olan veya olmayan bileşenlerin belirlenmesinde ve pek çok konuda oldukça etkili bir araç olan TSA yöntemi kullanılarak gerçekleştirilen ön işlem ve LSTM modeli ile gerçekleştirilen bir ileri tahmin çalışmasında MSE değeri 0.0021, MAE değeri 0.0361 ve R değeri 0.9710 olarak elde edilmiştir.

Elde edilen sonuçlar incelendiğinde TSA-LSTM modelinin performansının oldukça iyi olduğu TSA verilerin ön işlenmesi çalışmasının model performansını önemli derecede artırdığı görülmüştür. TSA-LSTM modelinin nehir akım tahmin çalışmalarında yüksek performanslı bir araç olarak kullanılabileceği görülmektedir. 


\section{Kaynakça}

[1] Bayazit M, (1998). Hidrolojik Modeller, İTÜ İnşaat Fakültesi Matbaası, İstanbul.

[2] Cuddington K., M. J. Forth, L. R. Gerber et al., "Process-based models are required to manage ecological systems in a changing world," Ecosphere, vol. 4, no. 2, pp. 1-12, 2013

[3] Liu, Z., Zhou, P., Chen, X., Guan, Y., 2015. A multivariate conditional model for streamflow prediction and spatial precipitation refinement. J. Geophys. Res. Atmos. 120 (19).

[4] Ekmekçi M. "Hacettepe Ders Notları",http://www.dsi.gov.tr/faaliyetler/turkiye-ulusal-hidroloji-komisyonu (Erişim tarihi, 2017)

[5] Box, G. E. P., Jenkins, G. M., 1970, Time Series Analysis: Forecasting and Control. Holden-Day, San Francisco.

[6] Carlson, R. F., MacCormick, A. J. A., Watts, D. G., 1970. "Application of linear models to four annual streamflow series", Water Resour. Res. 6 (4), 1070-1078.

[7] Zhang, H., Singh, V.P., Wang, B., Yu, Y., 2016. CEREF: A hybrid data-driven model for forecasting annual streamflow from a socio-hydrological system. J. Hydrol. 540, 246-256.

[8] Mehr A.D., 2018. "An improved gene expression programming model for streamflow forecasting in intermittent streams", Journal of Hydrology, 563, 669-678.

[9] Dehghani M., Seifi A., Madvar H.R., 2019 "Novel forecasting models for immediate-short-term to long-term influent flow prediction by combining ANFIS and grey wolf optimization", Journal of Hydrology, 576, 698-725.

[10] Sahoo A., Samantaray S., Ghose D. K., 2019. "Stream Flow Forecasting in Mahanadi River Basin using Artificial Neural Networks", Procedia Computer Science, 157, 168-174.

[11] He Y., Yan Y., Wang X., Wang C., 2019. "Uncertainty Forecasting for Streamflow based on Support Vector Regression Method with Fuzzy Information Granulation", Energy Procedia, 158, 6189-6194.

[12] Yu X., Wang Y., Wu L., Chen G., Wang L., Qind H., 2019. "Comparison of support vector regression and extreme gradient boosting for decomposition-based data-driven 10-day streamflow forecasting”, Journal of Hydrology, in press, 124293

[13] Hadi S. J., Tombul M., 2018. "Monthly streamflow forecasting using continuous wavelet and multi-gene genetic programming combination", Journal of Hydrology, 561, 674-687.

[14] Latifoğlu L., Kisi O., Latifoglu F., 2015. "Importance of hybrid models for forecasting of hydrological variable", Neural Computing \& Applications, 26, 1669-1680.

[15] Zealand CM., Burn DH., Simonovic SP., 1999, Short term streamflow forecasting using artificial neural networks, Journal of Hydrology, 214, 32-48

[16] Ni L., Wang D., Singh V. P., Wu J., Wang Y., Tao Y., Zhang J., 2019. "Streamflow and rainfall forecasting by two long shortterm memory-based models", Journal of Hydrology, in press, 124296.

[17] Mouatadid S., Adamowski J. F., Tiwari M. K., Quilty J. M., 2019. "Coupling the maximum overlap discrete wavelet transform and long short-term memory networks for irrigation flow forecasting", Agricultural Water Management, 219, 72-85.

[18] Dehghani M., Seifi A., Madvar H.R., 2019 "Novel forecasting models for immediate-short-term to long-term influent flow prediction by combining ANFIS and grey wolf optimization”, Journal of Hydrology, 576, 698-725.

[19] LeCun, Y., Bengio, Y., Hinton, G., 2015. Deep learning. nature 521 (7553), 436

[20] Ni L., Wang D., Singh V. P., Wu J., Wang Y., Tao Y., Zhang J., 2019. "Streamflow and rainfall forecasting by two long shortterm memory-based models", Journal of Hydrology, in press, 124296.

[21] Latifoğlu L., Kisi O., Latifoglu F., 2015. "Importance of hybrid models for forecasting of hydrological variable", Neural Computing \& Applications, 26, 1669-1680.

[22] Kisi O., Latifoğlu L., Latifoglu F., 2014. "Investigation of Empirical Mode Decomposition in Forecasting of Hydrological Time Series", Water Resources Management, 28, 4045-4057.

[23] Nourani V., Andalib G., Sadikoglu F., 2017. "Multi-station streamflow forecasting using wavelet denoising and artificial intelligence models", Procedia Computer Science, 120, 617-624.

[24] Fang W., Huang S., Ren K., Huang Q., Huang G., Cheng G., Li K., 2019. "Examining the applicability of different sampling techniques in the development of decomposition-based streamflow forecasting models", Journal of Hydrology, 568, 534-550.

[25] http://www.dsi.gov.tr/faaliyetler/akim-gozlem-yilliklari (Erişim Tarihi: Aralık 2019).

[26] Golyandina N., Zhigljavsky A., 2013, Singular Spectrum Analysis for Time Series, Springer.

[27] Sepp Hochreiter, Jurgen Schmidhuber, LONG SHORT-TERM MEMORY, Neural Computation, 9(8):1735-1780, 1997

[28] Olah, C. (2015, 27 Mayıs), Understanding LSTM Networks, Erişim Adresi: http://colah.github.io/posts/2015-08-UnderstandingLSTMs/ 